\title{
A BRIEF THEORY OVERVIEW OF HIGGS PHYSICS AT THE LHC*
}

\author{
Marius WiesemanN \\ Physik-Institut, Universität Zürich, 8057 Zürich, Switzerland
}

(Received October 19, 2015)

This is a brief review of the theoretical status of Higgs production at the LHC in the Standard Model, with an emphasis on the recent developments and computations. In particular, I focus on both inclusive and differential cross sections for the dominant production mode in the Standard Model, where the theoretical uncertainties induced by the different interplays between top- and bottom-quark effects in the gluon-fusion scattering amplitude are discussed.

DOI:10.5506/APhysPolB.46.2079

PACS numbers: 01.30.Cc, 14.80.Bn, 12.38.Bx

\section{Introduction}

The discovery of a scalar particle [1,2] is already considered as the legacy of the LHC Run 1. Several studies have analyzed the properties of this resonance (see, e.g., Refs. [3, 4]), all of which are fully consistent with the Standard Model (SM) picture. Such studies rely heavily on the precise theoretical understanding of the Higgs production and decay mechanisms.

Already with Run 1, but even more with the start of Run 2, the LHC is entering precision physics, in particular for Higgs related observables. This accuracy enables the measurement of differential distributions of the Higgs boson. First differential results were recently published in Refs. [5, 6]. With the additional precision expected from Run 2, such measurements will allow for the determination of small deviations from the SM picture in order to test the nature of the Higgs couplings. Therefore, accurate theoretical predictions are required not only for the total rate, but also for differential cross sections.

This contribution reviews the state-of-the-art predictions in the SM for the dominant gluon-induced Higgs production mode, where the total inclusive cross section as well as fully exclusive observables will be discussed.

* Presented at the XXXIX International Conference of Theoretical Physics "Matter to the Deepest", Ustroń, Poland, September 13-18, 2015. 


\section{Higgs production through gluon fusion}

Standard Model Higgs production proceeds predominantly via gluon fusion, where the Higgs-gluon coupling is mediated by a quark loop. Its cross section is roughly one order of magnitude larger than the sum of all other production modes. In the past years, gluon-induced Higgs production has become the theoretically best studied process at hadron colliders, which lead to a significant decrease of the related theoretical uncertainties.

An effective field theory approach, where the top quark is assumed to be infinitely heavy (also known as heavy-top limit), allows to determine higher order corrections roughly one perturbative order higher than in the full theory. One must bear in mind, however, that this approximation is strictly valid only when all scales remain below twice the top-quark mass. Therefore, care must be taken for the total inclusive cross section by estimating the effect of missing quark-mass contributions and for kinematical distributions outside the validity range of the approximation. In the latter case, the full theory must be employed at the cost of lower perturbative accuracy.

\subsection{Total inclusive cross section}

For a long time, the highest perturbative accuracy available for the total inclusive Higgs cross section in the infinite top-mass approximation was nextto-next-to-leading order (NNLO) [7-9]. Very recently, a milestone in perturbative computations was reached, when the first next-to-NNLO (N3LO) result at hadron colliders was computed [10] for this process. Similar to the NNLO result of Ref. [7], the computation of Ref. [7] employs a threshold expansion (including the first 39 terms) in $\tau=m_{H}^{2} / s$ around $\tau=1$, where $m_{H}$ is the mass of the Higgs boson and $s$ the partonic center-of-mass energy. While the leading terms of this expansion $[11,12]$ do actually not yet capture the dominant contribution of the N3LO corrections, the expansion stabilizes after $\sim 10$ terms [10]. This is indeed very similar to what was observed at NNLO [7], which later turned out to be in excellent agreement with the full result. There are essentially two reasons why the expansion works so well: soft-gluon effects close to the threshold are important, which are captured by this approach, and the gluon luminosities strongly suppress the region $z \ll 1$, where the expansion looses its validity. Hence, the computation of Ref. [10] can be safely considered to be the full N3LO result for all practical purposes.

The size of the N3LO corrections remains rather small $(\lesssim 1 \%)$, once a judicious choice for the renormalization and factorization scales of $m_{H} / 2$ at NNLO is made. This scale choice was motivated already by the good agreement with the soft gluon resummed result at NNLO+NNLL [13]. The actual benefit of the N3LO corrections is a significant reduction of the residual uncertainties leading to a prediction with a precision at the level of $2-3 \%$. 
Consequently, at N3LO the result is perturbatively highly stable, while all other uncertainties require now a careful estimate: PDF uncertainties are already of similar size at next-to-leading order (NLO) and at NNLO, which should not change at N3LO, since no dedicated N3LO PDF sets exist. They amount to typically $\sim 5 \%$ and have, therefore, become one of the major uncertainties on the cross section prediction for hadronic Higgs production at N3LO. Electroweak effects have been evaluated, for example, in Refs. [14-17]. The uncertainty induced by neglecting finite top-mass effects on the radiative corrections in the heavy-top approximation has been estimated to be below $1 \%$ for the total cross sections at NNLO by studying the asymptotic expansion in inverse powers of the top-quark mass $\left(1 / m_{\text {top }}^{2}\right)[18,19]$. One should bear in mind, however, that top mass effects must be fully accounted for at leading order and can then be supplemented by radiative corrections computed in the heavy-top approximation in terms of $K$-factors. For the partonic cross section, the $1 / m_{\text {top }}^{2}$ expansion reads

$$
\hat{\sigma}=\sum_{k=0}^{\infty} \frac{1}{m_{\mathrm{top}}^{2 k}} \hat{\sigma}^{(k)},
$$

which is strictly valid only for $\sqrt{s}<2 m_{\text {top }}$. Since the coefficients of this expansion actually diverge as $\sqrt{s} \rightarrow \infty$ [18], a matching of the partonic cross section to the high-energy limit $\hat{\sigma}(\sqrt{s} \rightarrow \infty)$ [18, 20] was introduced in Ref. $[18]^{1}$. The dependence of the estimated top-mass effects on the precise details of the matching procedure is negligible.

Concerning other quarks as mediators of the gluon-Higgs coupling, the effect of the four lightest quarks is $\lesssim 1 \%$. The bottom quark, on the other hand, contributes $\sim 5-10 \%$ to the total cross section at NLO [21]. Due to the small value of the bottom mass, a heavy-quark approximation as used for the top-quark contributions is not suitable in this case. Therefore, bottomquark effects must be included solely at the perturbative order, where the full quark-mass dependence on the cross section is known. Nevertheless, the uncertainty induced by the missing bottom-quark contributions at N3LO can simply be estimated by assuming their $K$-factor to be not larger than the one used for the top-quark contribution ${ }^{2}$. This leads to an estimate of at most $\pm 2-3 \%$ missing bottom-quark effects at N3LO.

In conclusion, the prediction for the total inclusive cross section for Higgs production through gluon fusion is under excellent theoretical control, which allows for actual precision physics at the LHC Run 2.

\footnotetext{
${ }^{1}$ Note that the region $\sqrt{s}>2 m_{\text {top }}$ is strongly suppressed by the gluon luminosities.

${ }^{2}$ Radiative corrections to the bottom loop are assumed to be smaller than for the top loop due to the softer spectrum. At NLO, this already turned out to be true.
} 


\subsection{Differential cross sections at fixed order}

Kinematical distributions in hadronic Higgs production provide an important handle on the determination of Higgs properties. Among the most relevant observables in this respect is the Higgs transverse momentum $\left(p_{\mathrm{T}}\right)$ spectrum. The NLO transverse momentum distribution of the Higgs boson in gluon fusion at $p_{\mathrm{T}}>0$ has been known for some time in the limit of heavy top quarks $[22,23]$. Recently, NNLO corrections to this observable were determined in a fully differential computation of the Higgs + jet rate [24-28]. Perturbative effects on the $p_{\mathrm{T}}$ spectrum at NNLO turn out to be quite sizable; they amount to $\sim 20 \%$ in the tail of the distribution.

A careful assessment of the validity of the heavy-top approximation becomes even more important for differential observables, where the hierarchy of different scales is more involved. Subleading top-mass effects on the differential Higgs + jet cross section at NLO have been considered in Refs. [29, 30]. Figure 1 shows the differential $K$-factor for the Higgs $p_{\mathrm{T}}$ distribution ${ }^{3}$

$$
K_{k}^{\mathrm{NLO}}\left(p_{\mathrm{T}}\right) \equiv\left[d \sigma^{\mathrm{NLO}} / d p_{\mathrm{T}}\right]_{m_{\mathrm{top}}^{k}} /\left[d \sigma^{\mathrm{LO}} / d p_{\mathrm{T}}\right]_{m_{\mathrm{top}}^{k}}
$$

as an expansion up to $1 / m_{\text {top }}^{k}$, where $k=0$ (dotted/green curve) corresponds to the heavy-top limit, $k=2$ (dashed/blue curve) involves terms up to $1 / m_{\text {top }}^{2}$ and $k=4$ (dash-dotted/yellow curve) includes the $1 / m_{\text {top }}^{4}$

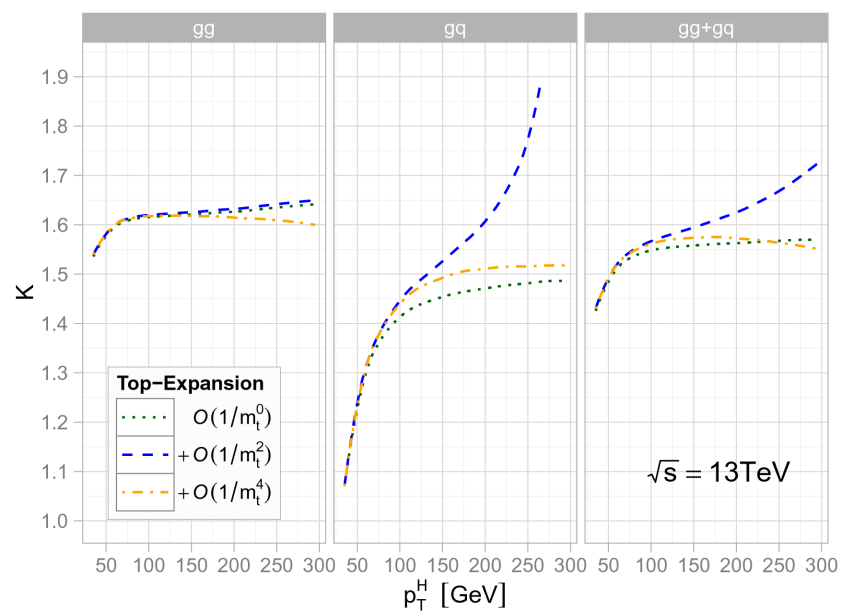

Fig. 1. $K$-factors for the Higgs $p_{\mathrm{T}}$ distribution as defined in Eq. (2), i.e. $K \equiv K_{k}^{\mathrm{NLO}}\left(p_{\mathrm{T}}\right)$. Left/center/right plot: only $g g /$ only $q g /$ sum of $g g$ and $q g$. Dotted/dashed/dash-dotted: $k=0 / 2 / 4$.

\footnotetext{
${ }^{3}[\ldots]_{m_{\mathrm{top}}^{k}}^{k}$ denotes the truncation of the asymptotic expansion at $1 / m_{\mathrm{top}}^{k}$.
} 
term in addition. The factorization and renormalization scales are set to the transverse mass of the Higgs boson $\mu_{\mathrm{F}}^{2}=\mu_{\mathrm{R}}^{2}=m_{H}^{2}+p_{\mathrm{T}}^{2}$. The left panel of Fig. 1 shows the purely gluon-induced subchannel, where the convergence of the asymptotic expansion is close to excellent at least for $p_{\mathrm{T}} \lesssim 150 \mathrm{GeV}$. This consistent picture deteriorates to some extent for the gluon-quark induced channel (central panel), but leads in the sum of both channels (right panel) to an overall reasonable convergence of the asymptotic series as long as $p_{\mathrm{T}} \lesssim m_{\text {top }}$. In that region, the uncertainty associated with missing topmass effects in the heavy-top limit can be estimated to be below $2-3 \%$, when taking the spread of the different curves as a measure of the size of the topquark effects. At $p_{\mathrm{T}}=300 \mathrm{GeV}$, the uncertainty is already at the level of $10 \%$ and the heavy-top limit starts to become unreliable.

It is obvious already from Fig. 1 that in the low- $p_{\mathrm{T}}$ region, the asymptotic expansion is well-behaved, while the convergence successively deteriorates as $p_{\mathrm{T}}$ (and therefore all associated scales) increases. Formally, any event with a hardness that exceeds the $2 m_{\text {top }}$ threshold is outside the validity range of the top-mass expansion. Considering the inclusive Higgs + jet cross section, where at least one hard jet is required ${ }^{4}$, the bulk of the well-behaved soft region is removed, and the problematic high-scale events are fully integrated over. For this observable, one, therefore, expects a badly converging asymptotic series, as can be seen from Fig. 2 (a). Indeed, none of the approximations agrees with the exact LO result (solid/red curve) and the ordinary $1 / m_{\text {top }}$ expansion, including the heavy-top limit, loses its predictive power.

However, one may recover the convergence of the asymptotic expansion, by introducing the matched Higgs + jet cross section as follows:

$$
\left[\sigma_{\geq 1-\text { jet }}^{\text {matched }}\right]_{m_{\mathrm{top}}^{k}} \equiv\left[\sigma_{\geq 1 \text {-jet }}^{\text {unmatched }}\right]_{m_{\mathrm{top}}^{k}}+\left[\sigma_{\mathrm{tot}}^{\text {matched }}\right]_{m_{\mathrm{top}}^{k}}-\left[\sigma_{\mathrm{tot}}^{\text {unmatched }}\right]_{m_{\mathrm{top}}^{k}} .
$$

As can be seen from Fig. 2 (b), the $1 / m_{\text {top }}^{2}$ series of the matched cross section is nicely convergent and in good agreement with the exact LO result at least for $p_{\mathrm{T}, \min }^{\text {jet }} \lesssim 150 \mathrm{GeV}$.

Equation (3) stems from the following observation: the total inclusive cross section $\sigma_{\text {tot }}$ is integrated over the same problematic high- $p_{\mathrm{T}}$ region as the Higgs + jet cross section. As stated before, a matching to the highenergy limit allows to control the region $\sqrt{s}>2 m_{\text {top }}$ in the case of the total cross section [18] (referred to $\sigma_{\text {tot }}^{\text {matched}}$ ). Assuming a similar matching for the inclusive Higgs + jet rate $\left(\sigma_{\geq 1 \text {-jet }}^{\text {matched }}\right)$, the difference between matched and unmatched cross sections for the total and the inclusive Higgs + jet rate should be the same up to a very good precision, as long as $p_{\mathrm{T}, \mathrm{min}}^{\mathrm{jet}}$ is chosen

${ }^{4}$ In this contribution, jets are defined using the anti- $k_{\mathrm{T}}$ algorithm [31] with jet radius $R=0.5$ and a requirement on the minimal jet transverse momentum of $p_{\mathrm{T}}^{\text {jet }}>p_{\mathrm{T}, \min }^{\text {jet }}$. 
at values below which the asymptotic expansion works well. This equality allows us to actually define the matched Higgs + jet cross section as done in Eq. (3). One must be careful, however, to combine the same orders in $\alpha_{\mathrm{s}}$ with a consistent set for the PDFs in that equation.

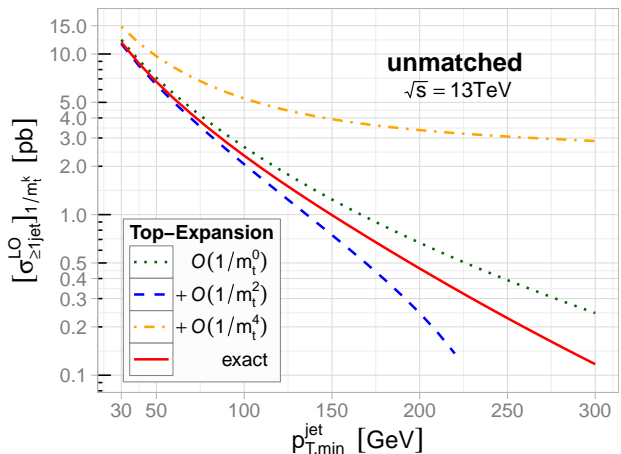

(a)

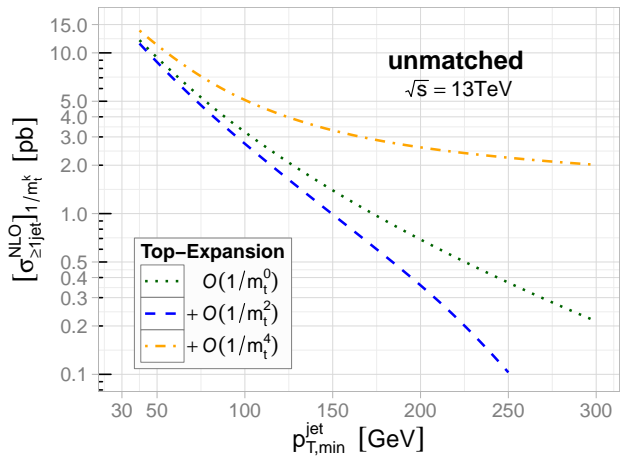

(c)

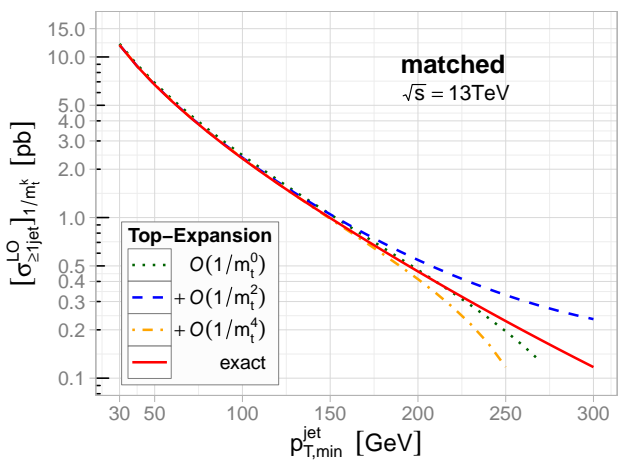

(b)

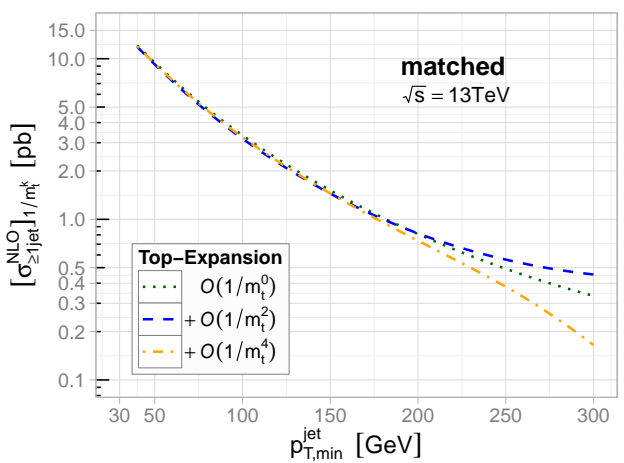

(d)

Fig. 2. Inclusive Higgs + jet rate at (a), (b) LO and (c), (d) NLO including terms up to $1 / m_{\mathrm{top}}^{k}$ as a function of $p_{\mathrm{T}, \min }^{\mathrm{jet}}$. Dotted/dashed/dash-dotted: $k=0 / 2 / 4$, solid: exact. (a), (c) unmatched; (b), (d) matched according to Eq. (3).

While at LO the exact result is known, the matched cross section proves particularly useful for the NLO Higgs + jet rate: Fig. 2 (c) shows a similarly bad convergence of the unmatched NLO Higgs + jet cross section as observed at LO. With the corresponding matching at $\alpha_{\mathrm{s}}^{4}$, shown in Fig. 2 (d), one obtains a nicely behaved asymptotic convergence at least for $p_{\mathrm{T}, \min }^{\text {jet }} \lesssim 150 \mathrm{GeV}$. In conclusion, the definition of the matched cross section enables a reliable prediction of the NLO Higgs + jet rate for standard experimental $p_{\mathrm{T}, \min }^{\text {jet }}$ cuts. 


\subsection{Differential cross sections with resummation}

It is well known that the perturbative ordering in $\alpha_{\mathrm{S}}$ breaks down in kinematical regions where logarithmically enhanced terms become large. One of such regions is $p_{\mathrm{T}} \ll Q$, where $Q \sim m_{H}$ is the typical hard scale of the Higgs production process. Only a resummation of logarithms in $p_{\mathrm{T}} / Q$ to all orders in $\alpha_{\mathrm{s}}$ provides a proper theoretical prediction. Such resummation can be performed analytically or by means of a parton shower (PS) approach.

Analytical transverse momentum resummation for the fusion process was calculated in the heavy-top approximation at next-to-next-to-leading logarithmic (NNLL) accuracy and consistently matched to the NNLO fixed-order cross section [32]. This computation is implemented in the publicly available programs HqT [32] and HRes [33, 34], where the latter is based on a Monte Carlo approach that allows for decays of the Higgs boson.

Finite top- and bottom-mass effects on the resummed $p_{\mathrm{T}}$ spectrum were discussed in Ref. [35] for the POWHEG [36] method at NLO+PS, in Refs. $[34,37,38]$ for analytic resummation through NLO+NLL and for MC@NLO [39] in Ref. [40]. While top-mass effects are moderate at small transverse momenta, sizable differences were originally observed between the POWHEG prediction for the combined top- and bottom-mass effects on the SM $p_{\mathrm{T}}$ spectrum and the other two approaches [35, 37, 40].

Common to these three approaches (analytic resummation, MCONLO, POWHEG) is an effective scale (resummation scale, shower scale, $h_{\text {fact }}$ ) that separates the soft/collinear from the hard region; referred to as matching scale in the following. Although the dependence on the matching scales is of higher logarithmic order, inadequate values may deteriorate the perturbative convergence due to large logarithms, which makes a careful choice necessary. The matching scale is set usually to the characteristic scale of the hard scattering process. However, if a process involves two or more different mass scales, as in the case of the bottom loop in Higgs production $\left(m_{\mathrm{b}}\right.$ and $m_{H}$ ), this choice is not at all obvious. Recently, two proposals were made for the algorithmic determination of suitable matching scales separately for the top, the bottom and the top-bottom interference contribution to the cross section [41, 42]. Indeed, for these matching scale choices, the differences observed in the three resummation/matching approaches at small $p_{\mathrm{T}}$ when including top- and bottom-mass effects are strongly reduced and the predictions become compatible within uncertainties (estimated from variations of the central matching scales by a factor of two) [43]. This is true not only in the SM, where the uncertainty induced by the bottom loop would not become too severe due to the small bottom Yukawa coupling, but also in extended models, where the coupling of the Higgs to bottom quarks can be significantly enhanced, and a careful choice of the matching scales becomes absolutely crucial [43]. 
Considering large transverse momenta, MCQNLO and analytic resummation are in good agreement due to their transition to the fixed-order prediction at high $p_{\mathrm{T}}$, while POWHEG develops a considerably larger cross section. The source of this enhanced tail is the particular treatment of multiple parton emissions by the PS, which acts on all transverse momenta in the ordinary POWHEG approach. However, it was shown [43] that a simple but powerful modification of the way POWHEG is interfaced to the PS leads to a consistent merging with the fixed-order prediction in the tail of the distribution and agreement with the other two approaches.

Regarding fully differential Monte Carlo predictions for hadronic Higgs production in the SM, a new generation of Monte Carlo tools has been developed in the recent past. These computations employ the highest perturbative information available in the heavy-top approximation and combine them with finite quark-mass effects. They can be divided into two classes: so-called NNLO+PS approaches [44-46], which merge Higgs plus zero and one jets at $\mathrm{NLO}+\mathrm{PS}$, while including NNLO corrections to the inclusive Higgs cross section and rapidity distribution; and NLO + PS merged computations of Higgs plus zero, one and two jets [47-50].

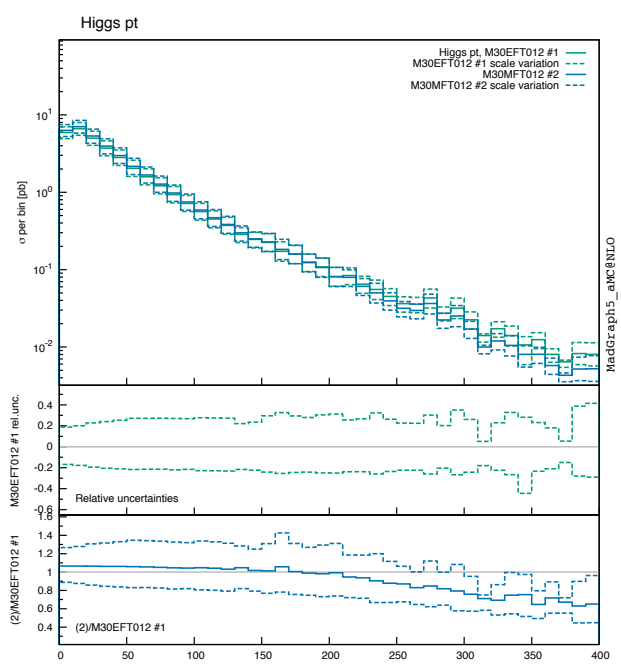

(a)

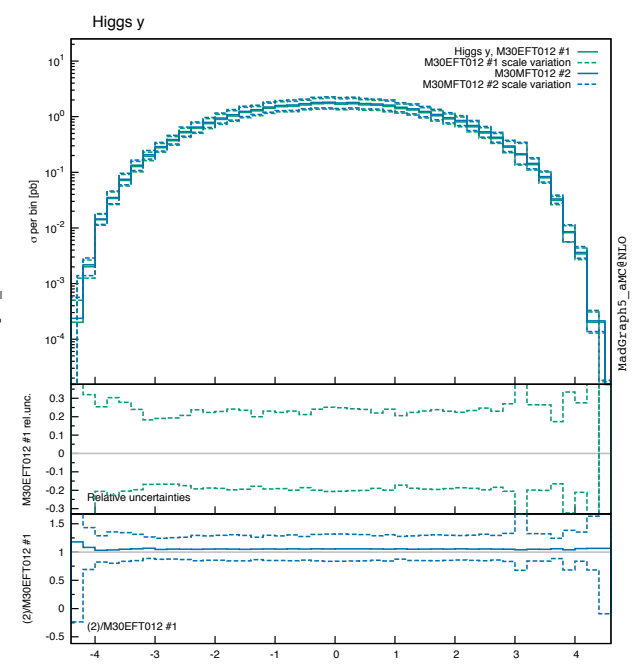

(b)

Fig. 3. (a) Higgs transverse momentum and (b) rapidity distribution in a merged NLO + PS Higgs plus zero, one, two jet computation. Solid light (green) curve: heavy-top limit; solid dark (blue) curve: including mass effects as described in the text. 
The currently most complete computation [48] in terms of heavy-quark mass effects employing all available exact matrix elements up to Higgs plus three jets, has been implemented by means of FxFx merging [47] in the MadGraph5_aMC@NLO framework [51]. The only approximated matrix elements are the two-loop virtuals for Higgs plus one and two jets, which are computed in the heavy-top limit and improved by reweighting them with the born amplitude in the full theory. Figure 3 compares the transverse momentum distribution (left panel) and rapidity distribution (right panel) of the Higgs boson in the heavy-top approximation (solid light (green) curve) to the prediction including top-mass effects (solid dark (blue) curve). From the ratio between the solid dark (blue) and the solid light (green) curve in the second inset, it is obvious that top-mass effects become particularly relevant at large transverse momentum scales and have a significant impact on the $p_{\mathrm{T}}$ shape, while for the rapidity distribution they essentially only affect the normalization. Bottom-mass effects can be added solely for Higgs plus zero jets at $\mathrm{NLO}+\mathrm{PS}$, since the heavy-quark approximation provides no adequate description in that case.

\section{REFERENCES}

[1] ATLAS Collaboration, Phys. Lett. B 716, 1 (2012).

[2] CMS Collaboration, Phys. Lett. B 716, 30 (2013).

[3] ATLAS Collaboration, Eur. Phys. J. C 75, 476 (2015).

[4] CMS Collaboration, Eur. Phys. J. C 75, 212 (2015).

[5] ATLAS Collaboration, Phys. Rev. Lett. 115, 091801 (2015).

[6] CMS Collaboration, arXiv:1508.07819 [hep-ex].

[7] R.V. Harlander, W.B. Kilgore, Phys. Rev. Lett. 88, 201801 (2002).

[8] C. Anastasiou, K. Melnikov, Nucl. Phys. B 646, 220 (2002).

[9] V. Ravindran, J. Smith, W.L. van Neerven, Nucl. Phys. B 665, 325 (2003).

[10] C. Anastasiou et al., Phys. Rev. Lett. 114, 212001 (2015).

[11] C. Anastasiou et al., Phys. Lett. B 737, 325 (2014).

[12] C. Anastasiou et al., arXiv:1411.3584 [hep-ph].

[13] D. de Florian, M. Grazzini, Phys. Lett. B 674, 291 (2009).

[14] G. Degrassi, F. Maltoni, Phys. Lett. B 600, 255 (2004).

[15] U. Aglietti, R. Bonciani, G. Degrassi, A. Vicini, Phys. Lett. B 595, 432 (2004).

[16] S. Actis, G. Passarino, C. Sturm, S. Uccirati, Phys. Lett. B 670, 12 (2008).

[17] C. Anastasiou, R. Boughezal, F. Petriello, J. High Energy Phys. 0904, 003 (2009).

[18] R.V. Harlander, H. Mantler, S. Marzani, K.J. Ozeren, Eur. Phys. J. C 66, 359 (2010). 
[19] A. Pak, M. Rogal, M. Steinhauser, J. High Energy Phys. 1109, 088 (2011).

[20] S. Marzani et al., Nucl. Phys. B 800, 127 (2008).

[21] M. Spira, A. Djouadi, D. Graudenz, P. Zerwas, Nucl. Phys. B 453, 17 (1995).

[22] D. de Florian, M. Grazzini, Z. Kunszt, Phys. Rev. Lett. 82, 5209 (1999).

[23] C.J. Glosser, C.R. Schmidt, J. High Energy Phys. 0212, 016 (2002).

[24] R. Boughezal et al., J. High Energy Phys. 1306, 072 (2013).

[25] X. Chen, T. Gehrmann, E.W.N. Glover, M. Jaquier, Phys. Lett. B 740, 147 (2015).

[26] R. Boughezal et al., Phys. Rev. Lett. 115, 082003 (2015).

[27] R. Boughezal et al., Phys. Lett. B 748, 5 (2015).

[28] F. Caola, K. Melnikov, M. Schulze, arXiv:1508.02684 [hep-ph].

[29] R.V. Harlander, T. Neumann, K.J. Ozeren, M. Wiesemann, J. High Energy Phys. 1208, 139 (2012).

[30] T. Neumann, M. Wiesemann, J. High Energy Phys. 1411, 150 (2014).

[31] M. Cacciari, G.P. Salam, G. Soyez, J. High Energy Phys. 0804, 063 (2008).

[32] G. Bozzi, S. Catani, D. de Florian, M. Grazzini, Nucl. Phys. B 737, 73 (2006).

[33] D. de Florian, G. Ferrera, M. Grazzini, D. Tommasini, J. High Energy Phys. 1206, 132 (2012).

[34] M. Grazzini, H. Sargsyan, J. High Energy Phys. 1309, 129 (2013).

[35] E. Bagnaschi, G. Degrassi, P. Slavich, A. Vicini, J. High Energy Phys. 1202 , 088 (2012).

[36] P. Nason, J. High Energy Phys. 0411, 040 (2004).

[37] H. Mantler, M. Wiesemann, Eur. Phys. J. C 73, 2467 (2013).

[38] A. Banfi, P.F. Monni, G. Zanderighi, J. High Energy Phys. 1401, 097 (2014).

[39] S. Frixione, B.R. Webber, J. High Energy Phys. 0206, 029 (2002).

[40] H. Mantler, M. Wiesemann, Eur. Phys. J. C 75, 257 (2015).

[41] R.V. Harlander, H. Mantler, M. Wiesemann, J. High Energy Phys. 1411, 116 (2014).

[42] E. Bagnaschi, A. Vicini, arXiv:1505.00735 [hep-ph].

[43] E. Bagnaschi et al., arXiv:1510.08850 [hep-ph].

[44] K. Hamilton, P. Nason, E. Re, G. Zanderighi, J. High Energy Phys. 1310, 222 (2013).

[45] S. Höche, Y. Li, S. Prestel, Phys. Rev. D 90, 054011 (2014).

[46] K. Hamilton, P. Nason, G. Zanderighi, J. High Energy Phys. 1505, 140 (2015).

[47] R. Frederix, S. Frixione, J. High Energy Phys. 1212, 061 (2012).

[48] R. Frederix, S. Frixione, E. Vryonidou, M. Wiesemann, to appear.

[49] S. Höche, F. Krauss, M. Schönherr, Phys. Rev. D 90, 014012 (2014).

[50] M. Buschmann et al., J. High Energy Phys. 1502, 038 (2015).

[51] J. Alwall et al., J. High Energy Phys. 1407, 079 (2014). 